\title{
Má rotação intestinal: um diagnóstico diferencial da doença do refluxo
}

\section{gastroesofágico}

\author{
Intestinal malrotation: a differential diagnosis of gastroesophageal reflux disease \\ Malrotación intestinal: un diagnóstico diferencial de la enfermedad por reflujo gastroesofágico
}

Recebido: 25/03/2021 | Revisado: 30/03/2021 | Aceito: 08/04/2021 | Publicado: 18/04/2021

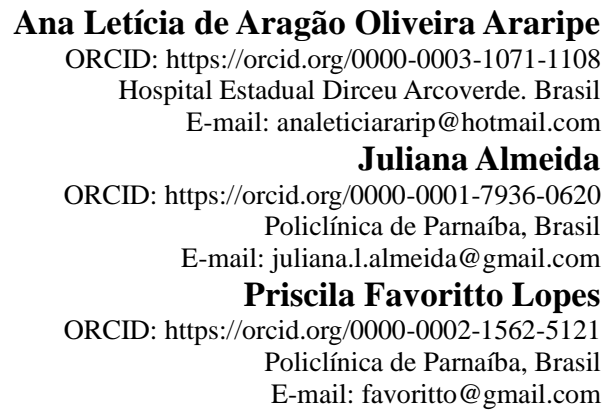

\begin{abstract}
Resumo
Neste relato iremos discorrer sobre um paciente de 7 anos de idade com diagnóstico de doença do refluxo gastroesofágico em tratamento desde os primeiros anos de vida. Apesar do tratamento clínico para a patologia, apresentava persistência de quadro de vômitos pós-alimentares precoces ocasionais, com necessidade de internação para compensação de distúrbios hidroeletrolíticos. Após ser encaminhado por um cirurgião, que suspeitou de necessidade de gastrofundoplicatura, o paciente procurou o serviço de urgência do Hospital Estadual Dirceu Arcoverde durante uma crise para ser avaliado pela Cirurgia Pediátrica. A hipótese de má rotação intestinal associada ou não a membrana duodenal foi prontamente aventada e investigada. Os exames de imagem corroboraram a suspeita e a cirurgia de Ladd foi realizada com sucesso. O relato foi elaborado através da análise de dados de prontuário de dois serviços médicos e fundamentado por pesquisa bibliográfica nas bases de dados Scielo e Pubmed. Nosso objetivo com este relato é trazer á memória um diagnóstico diferencial raro para uma sintomatologia compatível com patologia comum e salientar a importância do olhar de uma equipe multidisciplinar. Frente a um paciente com refluxo gastroesofágico, associado a déficit de crescimento e refratariedade no tratamento, diagnósticos diferenciais devem ser realizados e um deles é a má-rotação intestinal.
\end{abstract}

Palavras-chave: Refluxo gastroesofágico; Má rotação intestinal; Abdome agudo obstrutivo.

\begin{abstract}
This case report is about a 7 year old boy with gastroesophageal reflux disease (GERD) treating since his first years of life. Despite the adequate clinical treatment for this pathology, he occasionally presented with early post-prandial vomits, sometimes requiring hospital internment for hydro electrolyte disorder compensation. After being referred by a General Surgeon, who thought a fundoplication was needed, the patient went to the Dirceu Arcoverde Hospital during a vomiting crisis to be evaluated by a Pediatric Surgeon. The hypothesis of intestinal malrotation associated or not with duodenal web was promptly suggested. The image exams corroborated and Ladd's surgery was successfully performed. The case report was elaborated through the analysis of medical records of two medical services and reasoned by bibliographic research in the Scielo and Pubmed databases. Our goal with this paper is to bring to memory a differential diagnosis that is rare to a common pathology compatible group of symptoms and highlight the importance of a multidisciplinary team look. If exposed to a patient diagnosed with GERD, associated with growth stop of any kind and refractory clinical treatment, other diagnosis must be made and intestinal malrotation is one of them.
\end{abstract}

Keywords: Gastroesophageal reflux; Intestinal malrotation; Acute obstructive abdomen.

\section{Resumen}

En este relato vamos a discutir el caso de un paciente de 7 años com diagnostico de reflujo gastroesofagico en tratamiento desde los primeiros años de vida. Apesar del tratamiento clínico para la patología, presentava vomitos post-alimentares tempranos ocasionales, com necessidad de admisión hospitalar para control de disturbios hidroelectroliticos. Después de ser referenciado por um cirujano general, que sospechó de la necesidad de una fundoplicación, el paciente busco el servicio de urgencia del Hospital Estadual Dirceu Arcoverde durante uma crisis 
para evaluación de la Cirujía Pediátrica. La hipotesis de malrotación intestinal associada o no a uma membrana duodenal fue prontamente aventada e investigada. Los exámenes de imagen confirmaron la sospecha y la cirujia de Ladd fue realizada com éxito. El relato fue realizado por el analisis de datos de prontuário de dos servicios medicos y fundamentado por busca bibliografica en las bases de datos Scielo e Pubmed. Nuestro objetivo com este relato de caso es traer a la memoria um diagnostico diferencial incomun para sintomas compatibles com uma patologia muy frecuente y resaltar la importância de la evaluación de um equipo multidiciplinario. Frente a um paciente com enfermedad del reflujo gastroesofagico, presente em combinación com déficit de desarrollo y tratamiento refractario, nuevas hipotesis deven ser consideradas y una de ellas es la malrotacion intestinal.

Palabras clave: Reflujo gastroesofágico; Malrotación intestinal; Abdomen obstructivo agudo.

\section{Introdução}

O desenvolvimento intestinal é complexo e se inicia por volta da quinta semana de vida intrauterina, quando o intestino delgado começa um rápido processo de crescimento e excede a capacidade da cavidade abdominal, o que leva a uma herniação fisiológica temporária por volta da sexta semana. Durante este período, o intestino delgado sofre uma rotação antihorária de $270^{\circ}$ em torno do seu eixo, representado pela artéria mesentérica superior, que se completa por volta da décima semana, quando também se dá a redução da hérnia fisiológica (Devesa, 2015).

Durante o processo embriológico normal de rotação intestinal podem ocorrer algumas formas de anormalidades que constituem um espectro de condiç̃̃es referentes à má-rotação intestinal (Langer, 2017).

Em 30-60\% dos casos esta má-rotação pode associar-se a outras malformações e doenças como, por exemplo, atresia intestinal, intussuscepção, doença de Hirschsprung, anomalias das vias biliares, cardiopatia congênita, hérnia diafragmática congênita e defeitos de fechamento de parede abdominal (Cunha Filho, 2018).

O diagnóstico pré-natal da má-rotação intestinal isolada é difícil, entretanto após o nascimento, frente a quadro de vômitos de aspecto bilioso em recém-nascido previamente hígido, é necessário suspeitar imediatamente de obstrução intestinal neonatal e consequentemente de má rotação evoluindo com volvo de intestino médio (Holcomb, 2017). Até 75\% dos pacientes apresentam sintomas durante o primeiro mês de vida. Quando isto não ocorre pode-se ter maiores índices de atraso e erro diagnóstico, devido à variação de sintomas referidos que vão desde nenhum, até náuseas, vômitos recorrentes, dor abdominal, constipação e flatos, com curso crônico (Bezerra, 2019).

\section{Metodologia}

Relato de caso desenvolvido respeitando os princípios éticos presentes na declaração de Helsinque e de acordo com a Resolução 466/2012 do Conselho Nacional de Saúde (2013), que define as diretrizes e normas regulamentadoras de pesquisas, envolvendo principalmente seres humanos, bem como da carta circular 39/2011 que rege sobre utilização de dados secundários, assegurando sigilo dos mesmos (Conselho Nacional De Saúde, 2011). O presente trabalho trata de um estudo de caso qualitativo, que para Pereira, et al. (2018) é uma descrição bastante pormenorizada a qual pode suscitar uma riqueza de dados e informações levando à contribuição do saber na área de conhecimento que foi utilizado, de um paciente atendido e operado no Hospital Estadual Dirceu Arcoverde, acompanhado e investigado no Centro Integrado de Especialidades Médicas CIEM (fomentado pela FAPEPI) com má-rotação intestinal em novembro de 2020, cujos dados foram coletados através de registros em prontuário em ambos os serviços, análise de exames laboratoriais e de descrição cirúrgica, após assinatura, pela responsável da criança, do Termo de Consentimento Livre e Esclarecido. Para a discussão do caso foi realizada revisão de literatura sobre o tema abordado, através das bases de dados Scielo e PubMed. Na Scielo foram selecionados artigos livremente utilizando as palavras chave: refluxo gastroesofágico, má rotação intestinal e abdome agudo obstrutivo apenas com limitação de língua e artigos publicados nos últimos 10 anos e na Pubmed o termo de busca "gastroesophageal reflux" AND "intestinal malrotation" tendo como resultado 14 artigos. Após leitura de título e resumo foram eliminados 6 artigos e dos 
restantes, 2 eram muito antigos e não estavam disponíveis em meios eletrônicos.

\section{Relato do Caso}

Criança A. J. L. O., 7 anos, sexo masculino, compareceu ao hospital de referência em urgência e emergência da cidade de Parnaíba - PI, o Hospital Estadual Dirceu Arcoverde, acompanhado de sua mãe que relatou quadro de numerosos episódios de vômitos de aspecto bilioso, dificuldade em alimentar-se devido ao quadro de êmese e história de doença do refluxo gastroesofágico (DRGE) desde a primeira infância, para o qual fazia uso de medicação via oral. Nesta ocasião, que já era a terceira vez que buscava algum pronto socorro e que possuía necessidade de internação para correção de distúrbios hidroeletrolíticos. Apresentava-se com regular estado geral, consciente, orientado, acianótico, anictérico, hipocorado, desidratado, eupneico e com fáscies de dor. Após compensação clínica recebeu pedidos de exames de investigação e encaminhamento para seguimento ambulatorial com as equipes de Pediatria e Cirurgia Pediátrica na Policlínica de Parnaíba CIEM. Entretanto, antes de retornar com exames, apresentou novo episódio de distúrbio hidroeletrolítico com nova necessidade de permanência nosocomial. O único exame que conseguiu realizar neste interim foi endoscopia digestiva alta com biópsia, que revelou esofagite eosinofílica. Frente a tal diagnóstico e melhora clínica novamente com jejum de curto período e reposição eletrolítica, procedeu-se com tratamento clínico direcionado para esta patologia, sem sucesso.

Durante nova crise de vômitos com descompensação clínica o paciente foi internado, triado para avaliação nutricional e realizados exames de imagem para investigação de diagnósticos diferenciais. Foram realizados exames laboratoriais em que notamos que, apesar de o paciente ser emagrecido e ter o histórico relatado, possuía níveis de proteínas séricas com valores dentro da normalidade. Foi submetido também ao estudo do trânsito intestinal por meio de radiografias contrastadas que mostraram intensa dilatação gastroduodenal, importante gastroparesia e localização da transição duodeno-jejunal discretamente afilada e á direita da coluna vertebral e tomografia computadorizada de abdome que sugeriram fator obstrutivo extrínseco na região duodenal.

A partir disto optou-se por realizar uma laparotomia exploradora para melhor elucidação e resolução do quadro. Durante o procedimento cirúrgico, observou-se má-rotação de intestinal associada com banda de Ladd. O cólon direito se encontrava em topografia de epigástrio, sem fixação em retroperitônio e intensa distensão gastro-duodenal. Realizou-se então a cirurgia de Ladd.

O paciente, em seu pós-operatório imediato, ficou em observação e monitorização em leito de estabilização pediátrica, evoluindo com quadro clínico de gastroparesia que foi solucionada a contento, entretanto teve sua internação prolongada devido a quadro pulmonar compatível com pneumonia.

Durante seu seguimento ambulatorial relatou 2 episódios de dor abdominal intensa, com irradiação para o dorso, associados a vômitos que cessaram após analgesia comum e não houve necessidade de busca a serviços de urgência.

Ganhou 2 kilos de peso e a mãe relatou melhora da ansiedade por receber alimento que o paciente tinha antes da cirurgia.

Após 4 meses do procedimento de Ladd, logo após retorno ambulatorial, apresentou novo episódio de êmese persistente, de aspecto bilioso, associados a dor abdominal intensa refratária a analgesia comum, mais importante em topografia epigástrica, com irradiação para o dorso. Foi internado em hospital da sua cidade de origem e transferido após 3 dias para o HEDA. Foram realizados novos exames laboratoriais e de imagem, dentre eles, tomografia computadorizada de abdome, que revelou dilatação da câmara gástrica e imagem de rotação do mesentério ao nível do Treitz, mas sem outros sinais sugestivos de isquemia intestinal. Assim sendo, decidiu-se realizar nova laparotomia exploradora, onde foram encontradas bridas entre mesocólon e mesentério, com tunelização do jejuno no local do ângulo de Treitz. Novamente foi realizada a liberação de bandas na região do duodeno, que se encontrava com melhora importante mas ainda com distensão local, 
reforçada o alargamento do mesentério do intestino delgado e realizado o reposicionamento de duodeno e delgado á direita e ceco e cólons á esquerda. Após novo procedimento o paciente apresentou alta precoce com boa aceitação da dieta e compensação clínica em um período de 3 dias. Segue em seguimento ambulatorial, assintomático desde então.

\section{Resultados e Discussão}

A má rotação intestinal ocorre em aproximadamente $1 \%$ da população e na maior parte das vezes se manifesta com vômitos biliosos nos primeiros dias ou meses de vida. Já nesta fase o diagnóstico de refluxo esofágico se confunde quando a deterioração clínica secundária a uma torção do intestino médio não ocorre (El-Chammas, 2016). Após o primeiro ano de vida reduz muito a incidência de sintomas relacionadas á má rotação intestinal. São vagos e se confundem com diversas outras afecções, tais como causas psicogênicas, alergias alimentares, intolerância á lactose (Nagdeve, 2012). Mathews, Thenabadu e Jaiganesh (2011) acrescentam ainda mais diagnósticos diferenciais: pâncreas anular, intussuscepção intestinal, membrana duodenal, refluxo gastroesofágico, anemia e desnutrição.

O paciente relatado manifestava sintomas dispépticos desde a primeira infância, associados a vômitos biliosos pósalimentares em crises, os quais não exprimiam melhora mesmo em vigência de tratamento clínico para DRGE. Apresentava ainda déficit de ganho de peso, mantendo crescimento estatural, o que chama atenção, mas sugere que o quadro não é tão pronunciado assim.

Destarte, no relato apresentado os sintomas foram condizentes com os dados da literatura, entretanto, esta refere que a maioria dos casos de má rotação intestinal são diagnosticados principalmente no período neonatal ou ainda no primeiro ano de vida (Bezerra, et al., 2019; Emanwua, Ayantunde \& Davies, 2011), o que não ocorreu neste trabalho, haja vista que o quadro apenas foi descoberto aos 7 anos, o que tornou ainda mais importante seu diagnóstico diferencial com DRGE, já que na faixa etária pediátrica, esta apresenta-se classicamente por epigastralgia, pirose e sensação de plenitude gástrica (Sociedade Brasileira de Pediatria, 2017).

Em adultos o diagnóstico de má- rotação se torna ainda mais raro, e mais comumente se manifesta com sintomas crônicos e podem ser achado incidental durante outros procedimentos cirúrgicos.

A gastroparesia decorrente do quadro crônico de suboclusão intestinal determinado pela má rotação intestinal, frequentemente predispõe a ocorrência de refluxo gastroesofágico. A demora no esvaziamento gástrico estimula episódios de relaxamento do esfíncter esofágico inferior (Demirbilek, 2005). Estudos citados por Firat, Karakas \& Yakinci em 2007 mostram entre 57 a 94\% de associação entre as patologias citadas. Estes autores relatam um caso de Síndrome de Sandifer, um evento não epiléptico paroxístico clássicamente documentado nos pacientes com refluxo em recém-nascidos.

Apesar da correção do vício de rotação, Jolley, et al. em 1999 documentou refluxo persistente através da phmetria em 92\% dos casos após o procedimento de Ladd.

O paciente realizou alguns exames de imagem, dentre eles, tomografia computadorizada de abdome que evidenciou dilatação do estômago e porção proximal do duodeno, com imagem heterogênea mal delimitada ao nível da segunda porção do duodeno em situação paramediana direita, além de estudo do trânsito intestinal através de radiografias contrastadas cujos achados foram compatíveis com o quadro em estudo, visto que exibia a junção duodeno jejunal localizada à direita do corpo vertebral. Estes dados estão em conformidade com Langer (2017) o qual cita em seu trabalho que o padrão ouro para diagnóstico de má rotação intestinal é a radiografia contrastada gastrointestinal. O exame normal mostraria a junção duodenojejunal à esquerda do corpo vertebral, ao nível da margem inferior do bulbo duodenal em uma projeção anteroposterior e deve deslocar-se posteriormente na visão lateral. Dekonenko, et al. (2019) em seu estudo de 246 casos de um único serviço notou que, apesar deste consenso, frente ao aumento da idade dos pacientes, mais vezes a tomografia computadorizada de abdome foi solicitada em conjunto durante a investigação. 
O tratamento cirúrgico indicado no caso de má rotação é o procedimento de Ladd. Realizado em quatro etapas: liberação do duodeno, apendicectomia, lise de aderências entre mesentério e mesocólon e reposicionamento dos intestinos de forma que o intestino delgado se situe do lado direito do abdômen e o cólon do lado esquerdo (Davenport, 2020). Pode ser realizada por via laparoscópica ou laparotômica, como realizado no caso relatado. O trabalho de Isani, et al. de 2018 comparou ambas as vias de acesso e não detectou superioridade no tratamento lapascópico e sugere preferir a via aberta se a manifestação clínica for compatível com volvo.

Em sua revisão sistemática coletando dados de trabalhos sobre má rotação intestinal em adultos, Neville, Gallagher, Mitra e Sheth em 2020, viram que em alguns casos de achado incidental da patologia, a cirurgia de Ladd não foi realizada. Em parte, por falta de experiência dos cirurgiões gerais com este procedimento, em parte pelo fato de os pacientes não apresentarem sintomas crônicos relacionados á alteração encontrada. Além disso relataram que não há consenso sobre a indicação da cirurgia eletiva nos casos de pacientes assintomáticos.

Segundo Coe, Chang e Sicklick (2015) e Anand et al. (2018) ao diagnóstico tardio, esta condição tem sua morbidade intensificada, o que pode levar ao aumento de complicações perioperatórias e de reabordagem cirúrgica, corroborando com este relato onde o paciente apresentou quadro de gastroparesia e alterações pulmonares, compatíveis com pneumonia, após o primeiro procedimento cirúrgico e necessidade de reabordagem para desfazer novas bridas formadas entre mesocólon e mesentério 3 meses após a primeira cirurgia.

\section{Considerações Finais}

Conclui-se que a frente a patologias comuns que não respondem a um tratamento clínico prolongado, outras hipóteses diagnósticas devem sempre ser aventadas. O paciente do caso relatado procurou o serviço encaminhado por um cirurgião geral que acreditou que a refratariedade da doença do refluxo gastroesofágico era sinal de necessidade de gastrofundoplicatura. Ao avaliar exames antigos e ver que não havia esofagite compatível com um quadro de refluxo tão persistente, a suspeição do diagnóstico de uma má rotação, associada ou não a uma membrana duodenal, foi imediatamente levantada. Os exames de imagem confirmaram a hipótese e a cirurgia adequada possibilitou um ganho de qualidade de vida importante para paciente e família.

O diagnóstico de má rotação intestinal não faz parte do leque de patologias que estão constantemente na mente dos médicos da atenção primária, ou faz parte do dia a dia dos cirurgiões gerais. Ressalto, portanto, a importância do seguimento multidisciplinar dos pacientes e de manter sempre a mente aberta para os diagnósticos menos frequentes, para assim obtermos melhores resultados em nossa prática clínica.

\section{Referências}

Anand, U., Kumar, R., Priyadarshi, R. N., Kumar, B., Kumar, S., \& Singh, V. P. (2018). Comparative study of intestinal malrotation in infant, children, and adult in a tertiary care center in India. Indian journal of gastroenterology: official journal of the Indian Society of Gastroenterology, 37(6), 545-549. https://doi.org/10.1007/s12664-018-0914-1

Araújo, Ubirajara Rutilio Mendes e Ferreira de, \& El Tawil, Imad Izat. (2009). Má rotação intestinal em adulto, relato de caso e revisão da literatura. ABCD. Arquivos Brasileiros de Cirurgia Digestiva (São Paulo), 22(4), 240-242. https://doi.org/10.1590/S0102-67202009000400013

Coe, T. M., Chang, D. C., \& Sicklick, J. K. (2015). Small bowel volvulus in the adult populace of the United States: results from a population-based study. American journal of surgery, 210(2), 201-210.e2. https://doi.org/10.1016/j.amjsurg.2014.12.048

Comissão Nacional de Ética em Pesquisa (2011). Carta Circular. nº 039/2011, de 20 de setembro de 2011. Distrito Federal: Conselho Nacional de Saúde: https://conselho.saude.gov.br/Web_comissoes/conep/carta_circular/Uso_de_dados_de_prontuarios_para_fins_de_Pesquisa.pdf.

Conselho Nacional de Saúde. (2013). Resolução n 466/12, de 12 de dezembro de 2012. Diário Oficial da União: seção 1, (12) 59.

Cunha Filho, A. A. A., Coimbra, P. A., Perez-Bóscolo A. C., Dutra, R. A., \& Alves, K. P. O.(2018) Má-rotação intestinal: um diagnóstico a ser considerado no abdome agudo em recém-nascidos. Residência Pediátrica 8(3):141-146. https://doi.org/10.25060/residpediatr-2018.v8n3-08 
Research, Society and Development, v. 10, n. 4, e49010414276, 2021

(CC BY 4.0) | ISSN 2525-3409 | DOI: http://dx.doi.org/10.33448/rsd-v10i4.14276

Davenport, M., Geiger, J., Hall, N., \& Rothenberg, S. (2020) Operative pediatric surgery (8a ed.). Boca Raton: CRC Press.

Dekonenko, C., Sujka, J. A., Weaver, K., Sharp, S. W., Gonzalez, K., \& St Peter, S. D. (2019). The identification and treatment of intestinal malrotation in older children. Pediatric surgery international, 35(6), 665-671. https://doi.org/10.1007/s00383-019-04454-9

Demirbilek, S., Karaman, A., Gürünlüoğlu, K., Akin, M., Taş, E., Aksoy, R. T., \& Kekilli, E. (2005). Delayed gastric emptying in gastroesophageal reflux disease: the role of malrotation. Pediatric surgery international, 21(6), 423-427. https://doi.org/10.1007/s00383-005-1460-3

Devesa, H., Lima, B., Martins, A., Ferreira, M., Vasconcelos, E., Torres, R., \& Midões, A. (2015). Obstrução intestinal alta por Bandas de Ladd. Revista Portuguesa De Cirurgia, (34), 49-54. Acesso em de https://revista.spcir.com/index.php/spcir/article/view/544

El-Chammas, K., Malcolm, W., Gaca, A. M., Fieselman, K., \& Cotten, C. M. (2006). Intestinal malrotation in neonates with nonbilious emesis. Journal of perinatology: official journal of the California Perinatal Association, 26(6), 375-377.

Emanuwa, O. F., Ayantunde, A. A., \& Davies, T. W. (2011). Midgut malrotation first presenting as acute bowel obstruction in adulthood: a case report and literature review. World journal of emergency surgery: WJES, 6(1), 22. https://doi.org/10.1186/1749-7922-6-22

Firat, A. K., Karakas, H. M., Firat, Y., \& Yakinci, C. (2007). Unusual symptom of intestinal malrotation: episodic cervical dystonia due to Sandifer syndrome. Pediatrics international: official journal of the Japan Pediatric Society, 49(4), 519-521. https://doi.org/10.1111/j.1442-200X.2007.02409.x

Holcomb, G. W., Murphy, J. P., Ostlie, D. J. (2017) Holcomb and Ashcraft's Pediatric Surgery. (6a ed.), Elsevier.

Isani, M. A., Schlieve, C., Jackson, J., Elizee, M., Asuelime, G., Rosenberg, D., Kim, E.S. (setembro de 2018) Is less more? Laparoscopic versus open Ladd's procedure in children with malrotation. Journal of surgical research, 229, 351-356. https://doi.org/10.1016/j.jss.2018.04.016

Langer J. C. (2017). Intestinal Rotation Abnormalities and Midgut Volvulus. The Surgical clinics of North America, 97(1), 147-159. https://doi.org/10.1016/j.suc.2016.08.011

M., \& Kurlinski, J. P. (1999). Esophageal pH monitoring abnormalities and gastroesophageal reflux disease in infants with intestinal malrotation. Archives of surgery (Chicago, Ill: 1960), 134(7), 747-753. https://doi.org/10.1001/archsurg.134.7.747

Mathews, R., Thenabadu, S., \& Jaiganesh, T. (2011). Abdominal pain with a twist. International journal of emergency medicine, 4, 21. https://doi.org/10.1186/1865-1380-4-21

Nagdeve, N. G., Qureshi, A. M., Bhingare, P. D., \& Shinde, S. K. (2012). Malrotation beyond infancy. Journal of pediatric surgery, 47(11), $2026-2032$. https://doi.org/10.1016/j.jpedsurg.2012.06.013

Neville, J. J., Gallagher, J., Mitra, A., \& Sheth, H. (2020). Adult Presentations of Congenital Midgut Malrotation: A Systematic Review. World journal of surgery, 44(6), 1771-1778. https://doi-org.ez17.periodicos.capes.gov.br/10.1007/s00268-020-05403-7

Pereira A. S. et al. (2018). Metodologia da pesquisa científica. UFSM. https://repositorio.ufsm.br/bitstream/handle/1/15824/Lic_Computacao_MetodologiaPesquisa-Cientifica.pdf?sequence $=1$

Sociedade Brasileira de Pediatria (2017, dezembro) Regurgitação do Lactente (Refluxo Gastroesofágico Fisiológico) e Doença do Refluxo Gastroesofágico em Pediatria. Documento científico; Departamento Científico de Gastroenterologia. n. 2. 